\title{
spfilteR: An R package for Semiparametric Spatial Filtering with Eigenvectors in (Generalized) Linear Models
}

by Sebastian Juhl

\begin{abstract}
Eigenvector-based Spatial filtering constitutes a highly flexible semiparametric approach to account for spatial autocorrelation in a regression framework. It combines judiciously selected eigenvectors from a transformed connectivity matrix to construct a synthetic spatial filter and remove spatial patterns from model residuals. This article introduces the spfilte $\mathbb{R}$ package that provides several useful and flexible tools to estimate spatially filtered linear and generalized linear models in $R$. While the package features functions to identify relevant eigenvectors based on different selection criteria in an unsupervised fashion, it also helps users to perform supervised spatial filtering and to select eigenvectors based on alternative user-defined criteria. Besides a brief discussion of the eigenvector-based spatial filtering approach, this article presents the main functions of the package and illustrates their usage. Comparison to alternative implementations in other $\mathrm{R}$ packages highlights the added value of the spfilteR package.
\end{abstract}

\section{Introduction}

The presence of spatial autocorrelation in regression residuals constitutes a severe problem in standard inferential statistics as it causes common econometric methods to produce inefficient or even biased and inconsistent parameter estimates (Darmofal, 2015; Goodchild, 2009; Franzese and Hays, 2007). Besides parametric spatial regression techniques, which became the dominant approach to this challenge in the social sciences, spatial filtering techniques offer an alternative approach to handle spatially clustered data. The particular appeal of these alternative semiparametric approaches to spatial autocorrelation arise from their flexibility and the relative ease of estimation and interpretation (e.g., Tiefelsdorf and Griffith, 2007; Getis and Griffith, 2002). Especially the eigenvector-based spatial filtering (ESF) approach pioneered by Griffith $(2003,2000,1996)$ has proven to be useful in various academic disciplines.

This article introduces the spfilteR package that provides a set of flexible and useful functions to implement the ESF approach in regression models. Besides tools to detect spatial autocorrelation in individual variables and regression residuals by means of the Moran coefficient (MC) (Cliff and Ord, 1981, 1972), the package features easily customizable functions which allow users to perform supervised and unsupervised spatial filtering with eigenvectors. While other $\mathrm{R}$ packages like spatialreg (Bivand and Piras, 2015) and spmoran (Murakami, 2020) also contain implementations of the unsupervised ESF approach, they are less flexible in the specification of eigenvector selection criteria which constitutes the crucial step in the ESF approach. These packages also offer few functions for the supervised selection of eigenvectors.

In contrast, the spfilteR package allows users to obtain eigenvectors from a transformed connectivity matrix and to identify a suitable candidate set in order to perform supervised spatial filtering. Alternatively, unsupervised eigenvector selection procedures for different (generalized) linear models based on a stepwise regression procedure are implemented as well. These functions select eigenvectors based on either i) the maximization of model fit, ii) minimization of residual autocorrelation, iii) the statistical significance of residual autocorrelation, or iv) the statistical significance of the candidate eigenvectors. Parameter estimates are obtained by means of ordinary least squares (OLS) for linear models and maximum likelihood estimation (MLE) for generalized linear models (GLMs). The print, summary, and plot methods further facilitate the interpretation and visualization of the results.

After a theoretical description of the ESF approach in a regression framework, this article presents some stylized R code to demonstrate the implementation of the ESF approach using the functions and the synthetic dataset accompanying the spfilteR package. It also briefly compares the unsupervised ESF procedures contained in this package to alternative implementations in other $\mathrm{R}$ packages. The last section summarizes and concludes this article.

\section{Spatial filtering with eigenvectors}

Intuitively, the ESF approach put forth by Griffith $(2003,2000,1996)$ and also Tiefelsdorf and Griffith (2007) addresses the problem of spatially autocorrelated regression residuals by partitioning the error 
term into a spatially structured and a random component (see also Griffith and Chun, 2014). Consider a stylized linear regression model of the following form:

$$
y=X \beta+e,
$$

where $X$ denotes the matrix of covariates (plus a vector of ones for the intercept term) and $\beta$ is the corresponding parameter vector. If the errors $e$ are not independent but exhibit a non-random spatial pattern, the ESF approach removes this pattern from the disturbances and thereby "whitens" the residuals.

To this end, synthetic proxy variables are generated that reflect the spatial pattern present in model residuals as closely as possible. Subsequently including these synthetic variables as control variables in the regression's mean equation removes the problematic spatial structure from the disturbances and allows the use of standard procedures — such as OLS or MLE - for parameter estimation. Generating these proxy variables that act as the spatial filter requires the decomposition of the transformed and exogenously defined connectivity matrix which represents the dependence structure among the units of analysis.

\section{Eigenfunction decomposition}

The eigenfunction (or spectral) decomposition of a transformed connectivity matrix constitutes the core element of the ESF approach. More formally, the decomposition yields

$$
M V M=E \Lambda E^{\prime},
$$

where $\boldsymbol{M}$ is a symmetric and idempotent projection matrix, and $\boldsymbol{V}$ is the exogenously specified connectivity matrix which is symmetrized by $\frac{1}{2}\left(\boldsymbol{W}+\boldsymbol{W}^{\prime}\right)$. The columns of matrix $\boldsymbol{E}$ are the $n$ mutually uncorrelated eigenvectors obtained from $\boldsymbol{M V} \boldsymbol{M}$, and $\Lambda$ is a diagonal matrix with the corresponding eigenvalues $\lambda=\left\{\lambda_{1}, \lambda_{2}, \ldots, \lambda_{n}\right\}$ on its main diagonal. Tiefelsdorf and Boots (1995) show that each eigenvector in $E$ represents a distinct map pattern permitted by the units' spatial arrangement and is associated with a certain level of spatial autocorrelation. ${ }^{1}$

The projection matrix is given by $\boldsymbol{M}=\boldsymbol{I}-\boldsymbol{X}\left(\boldsymbol{X}^{\prime} \boldsymbol{X}\right)^{-1} \boldsymbol{X}^{\prime}$, where $\boldsymbol{I}$ is the identity matrix, and the eigenvectors in $E$ are mutually uncorrelated and orthogonal to the covariates in the design matrix $\boldsymbol{X}^{2}{ }^{2}$ If only the intercept is included in the construction of the projection matrix, this equation simplifies to $\boldsymbol{M}=\left(\boldsymbol{I}-\mathbf{1 1}^{\prime} / n\right)$, where $\mathbf{1}$ is an $n \times 1$-dimensional vector of ones. As Tiefelsdorf and Griffith (2007) show, the underlying spatial process generating the data determines both the form of the spatial misspecification in a naïve nonspatial regression and the appropriate specification of $\boldsymbol{M}$.

However, since the number of eigenvectors equals the number of observations in the data, only a subset of eigenvectors can be included in the regression equation.

\section{Eigenvector selection and the spatial filter}

Identifying and selecting relevant eigenvectors is decisive in the ESF approach and involves two steps. In a first step, a set of candidate eigenvectors, the search set $E^{C} \subset E$, needs to be determined based on different criteria. If the model residuals exhibit positive levels of spatial autocorrelation, eigenvectors depicting negative autocorrelation can be discarded since simultaneously including eigenvectors associated with positive and negative spatial autocorrelation can cause problems (Tiefelsdorf and Griffith, 2007). Moreover, eigenvectors portraying negligible levels of spatial autocorrelation can be eliminated as well since they contribute little to the spatial pattern present in model residuals (Chun and Griffith, 2014).

Griffith (2003), for example, proposes a qualitative threshold determining the candidate set by computing $M C_{i} / M C_{\text {max }}$ for all eigenvectors $i \in\{1,2, \ldots, n\}$, where $M C_{\text {max }}$ denotes either the largest positive or the largest negative Moran coefficient of all eigenvectors in $E$. According to this approach, eigenvectors for which $M C_{i} / M C_{\text {max }} \geq 0.25$ should be included in the candidate set $\boldsymbol{E}^{C}$. Alternatively, Chun et al. (2016) propose a nonlinear function to calculate the ideal size of the candidate set for a given level of spatial autocorrelation and the total number of positive eigenvectors. However, this approach is only applicable if the residuals exhibit positive levels of spatial autocorrelation.

\footnotetext{
${ }^{1}$ The Moran coefficient for each eigenvector in $\boldsymbol{E}$ can be computed by $M C_{i}=\lambda_{i} n / \mathbf{1}^{\prime} \boldsymbol{V} \mathbf{1}$ (e.g., Griffith, 1996; Tiefelsdorf and Boots, 1995).

${ }^{2}$ It is important to note that, just like weights in linear models, the presence of a link function corrupts the uncorrelatedness of the eigenvectors in generalized linear models (e.g., Griffith, 2003, 104-105). To check for problematic levels of multicollinearity among the eigenvectors, the function glmFilter () in the spfilteR package reports the condition number (see also Griffith and Amrhein, 1997).
} 
Once a feasible candidate set is identified, the importance of each eigenvector in $E^{C}$ needs to be established in a second step. This is typically done by a stepwise regression procedure which sequentially evaluates each eigenvector in the candidate set. To this end, the search algorithm utilizes an objective function in order to determine which eigenvectors to select. The selected eigenvectors $E^{*} \in E^{C}$ are the synthetic covariates constituting the spatial filter. Selection criteria commonly employed in the literature include the maximization of model fit statistics (e.g., Tiefelsdorf and Griffith, 2007; Griffith, 2003), the statistical significance of the eigenvectors (e.g., Griffith and Chun, 2014; Le Gallo and Páez, 2013), the minimization of residual autocorrelation (e.g., Tiefelsdorf and Griffith, 2007), or arbitrary combinations of different selection criteria (e.g., Páez, 2019). The aim is to specify an objective function that provides a parsimonious subset of eigenvectors. Parsimony here means that $E^{*}$ minimizes residual autocorrelation with respect to the pre-specified connectivity structure of the filtered model by selecting the smallest number of eigenvectors possible to obtain spatially independent errors (Tiefelsdorf and Griffith, 2007).

Once $\boldsymbol{E}^{*}$ is established, it can be added to the regression model in Equation (1):

$$
y=X \beta+\overbrace{\underbrace{E^{*} \gamma}_{\text {filter }}+\underbrace{\epsilon}_{\text {noise }}}^{e} .
$$

Equation (3) depicts the spatially filtered regression model and illustrates how the ESF approach partitions the regression residuals $\boldsymbol{e}$ from Equation (1) into a spatial trend component $\left(E^{*} \gamma\right)$ and a random component $(\epsilon)$. The selected eigenvectors $E^{*}$, in conjunction with their parameter estimates $\gamma$, represent the spatial pattern latent in $\boldsymbol{e}$. This term constitutes the synthetic spatial filter that shifts the spatial pattern from the error term to the regression's systematic part. Thereby, it removes the spatial structure from the error term, leaving white noise residuals $\epsilon$.

This stylized filtering scheme directly extends to GLMs, although the link function might corrupt the uncorrelatedness of the eigenvectors. If a substantial amount of multicollinearity among the eigenvectors is present, each eigenvector included in the subset of $E^{*}$ should be reevaluated whenever a new eigenvector is selected (e.g., Griffith et al., 2019).

\section{The spfilteR package}

The stable release version of the spfilteR package can be obtained from CRAN. ${ }^{3}$ Alternatively, the latest development version is available on GitHub:

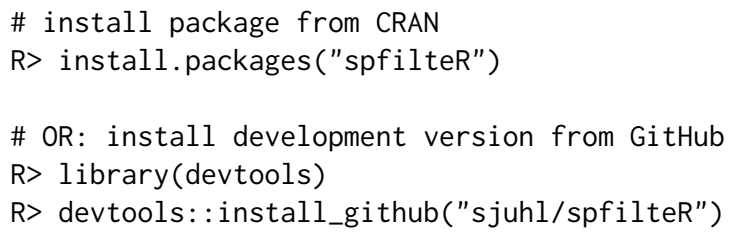

Alongside a collection of functions, the package also provides an artificial dataset and a stylized binary connectivity matrix based on the rook scheme of adjacency that connects $n=100$ units on a regular $10 \times 10$ grid. I use this made-up dataset to illustrate key features of the package and its functionality.

To this end, consider a simple linear regression model with a single regressor. Once the model is fitted, the function MI . resid() performs a test of residual spatial autocorrelation based on the Moran coefficient (Cliff and Ord, 1981).

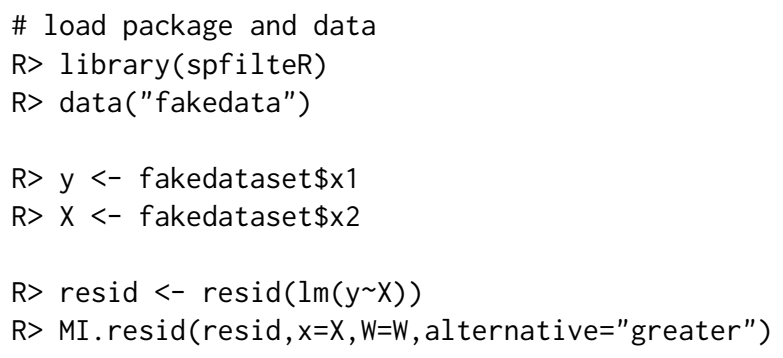

${ }^{3}$ This article is based on version 1.0 .0 of the spfilteR package. 
$\begin{array}{rrrrr}I & E I & \operatorname{VarI} & \text { zI } & \text { pI } \\ 0.350568 & -0.0119261 & 0.01207299 & 3.299085 & 0.0004850019\end{array} * * *$

The results suggest that the residuals are spatially autocorrelated, which violates the Gauss-Markov assumption of uncorrelated errors since $\operatorname{cov}\left(\epsilon_{i}, \epsilon_{j}\right) \neq 0 \forall i \neq j$. To address this problem, the spfilteR package allows users to implement the ESF approach and to select relevant eigenvectors using different supervised or unsupervised selection procedures.

\section{Supervised spatial filtering}

As shown above, the ESF approach starts with the eigenfunction decomposition of a transformed and symmetrized connectivity matrix as depicted in Equation (2). The function getEVs() allows users to easily obtain these eigenvectors. Moreover, users have the option to specify covariates that are used in order to construct the projection matrix $M$ via the input covars.

R $>$ EVs $<-$ getEVs $(W=W$, covars $=$ NULL $)$

$R>E<-E V s \$$ vectors

In addition to the eigenvectors and their corresponding eigenvalues, getEVs () also reports the value of the MC associated with each of the eigenvectors. ${ }^{4}$ The first eigenvector depicts the spatial pattern permitted by $W$ with the largest possible degree of positive spatial autocorrelation. The second eigenvector displays the pattern associated with the second largest possible degree of positive autocorrelation that is uncorrelated with the first pattern, and so on (Griffith, 1996). Consequently, while the first eigenvectors represent global patterns of positive spatial autocorrelation, the pattern becomes more local as the degree of spatial autocorrelation approaches zero. The last eigenvectors in the set capture patterns of negative autocorrelation (see Figure 1).
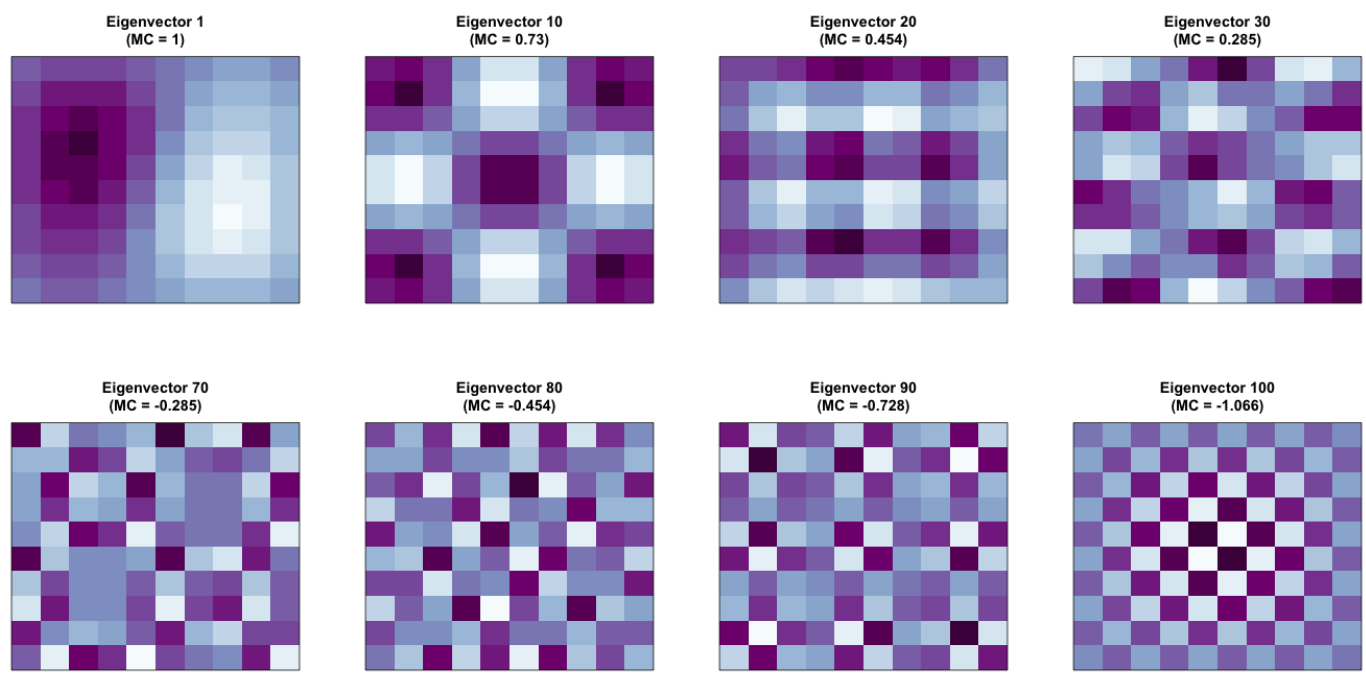

Figure 1: Visualization of Eigenvectors and their respective Moran coefficient (MC). Positive spatial patterns are shown in the first row while negative patterns are depicted in the second row.

Based on the MC values, users can define the candidate set $E^{C}$ and select relevant eigenvectors based on any desired selection criterium. Using the threshold suggested by Griffith (2003) outlined above, the set $E^{C}$ consists of 31 eigenvectors. For illustrative purposes, I skip the second step of the eigenvector selection procedure and include all eigenvectors in the ESF model so that $E^{C}=E^{*}$.

\# identify candidate set

$\mathrm{R}>\mathrm{Ec}<-\mathrm{EV}$ s\$moran/max $(\mathrm{EV}$ s $\$$ moran $)>=.25$

\# obtain ESF residuals

$\mathrm{R}>$ esf.resid <- $\operatorname{resid}(\operatorname{lm}(\mathrm{y} \sim \mathrm{X}+\mathrm{E}[, \mathrm{Ec}]))$

\# check for remaining spatial autocorrelation in model residuals

R> MI.resid(esf.resid, $\mathrm{x}=\mathrm{X}, \mathrm{W}=\mathrm{W}$, alternative="greater")

\footnotetext{
${ }^{4}$ To this end, getEVs () calls the helper function MI.ev(), which calculates the MC for each supplied eigenvector (see also Griffith, 1996; Tiefelsdorf and Boots, 1995).
} 


$\begin{array}{rrrrr}I & E I & V a r I & \text { zI } & \text { pI } \\ -0.1836998 & -0.0119261 & 0.01207299 & -1.563326 & 0.941012\end{array}$

The results indicate that the ESF approach successfully removed positive spatial autocorrelation from regression residuals. Furthermore, the functions partialR2() and vif.ev() included in the spfilteR package allow users to investigate the proportion of explained variance by each eigenvector and identify potential problems of variance inflation. In this example, eigenvector 13 accounts for about $23.21 \%$ of the variance in $y$. Moreover, none of the eigenvectors induces problematic levels of multicollinearity as the variance inflation factor (VIF) of each eigenvectors remains close to 1 .

$R>\operatorname{round}(\operatorname{partialR} 2(y=y, x=X, \operatorname{evecs}=E[, E c]), 6)$

$\begin{array}{llllllllll}0.000377 & 0.060584 & 0.001004 & 0.028734 & 0.020554 & 0.004804 & 0.000091 & 0.007010\end{array}$

$\begin{array}{lllllllllll}0.030418 & 0.079015 & 0.004550 & 0.000012 & 0.232083 & 0.011407 & 0.000959 & 0.004993\end{array}$

$\begin{array}{lllllllll}0.001714 & 0.000094 & 0.036713 & 0.044113 & 0.006588 & 0.005762 & 0.001845 & 0.009648\end{array}$

$\begin{array}{lllllll}0.002761 & 0.031923 & 0.007490 & 0.000075 & 0.004271 & 0.004042 & 0.004060\end{array}$

R> vif.ev $(x=X$, evecs $=E[, E c], n a . r m=T R U E)$

1.0044201 .0016601 .0504091 .0497291 .0118991 .0015881 .0083931 .000929

$\begin{array}{llllllll}1.034209 & 1.013360 & 1.000230 & 1.000027 & 1.005781 & 1.022793 & 1.073397 & 1.015425\end{array}$

$\begin{array}{lllllllll}1.014602 & 1.014900 & 1.000798 & 1.002998 & 1.004616 & 1.019448 & 1.001397 & 1.015900\end{array}$

1.0055401 .0004741 .0183441 .0083631 .0002841 .0097561 .086114

\section{Unsupervised spatial filtering}

Besides the supervised eigenvector selection procedure, the function lmFilter() performs unsupervised spatial filtering and provides parameter estimates by means of OLS. Importantly, users can specify different selection criteria. Thereby, this function eases the implementation of the ESF approach while simultaneously providing considerable flexibility regarding the stepwise selection of eigenvectors. Specifically, the following input arguments allow users to customize the selection procedure and ensure the function's flexibility:

- objfn allows users to determine the objective function of the search algorithm determining $E^{*}$. It supports eigenvector selection based on the adjusted $R^{2}$ ('R2'), residual spatial autocorrelation ('MI '), the significance of eigenvectors ( $P$ ' ), and the significance level of residual spatial autocorrelation (' pMI '). Alternatively, all eigenvectors may be included by spefifying objfn=' all' , implying that no selection takes place.

- MX (optional) specifies the covariates used to construct the projection matrix $M$. As Tiefelsdorf and Griffith (2007) show, the specification of $\boldsymbol{M}$ is directly linked to the form of the spatial misspecification in the unfiltered naïve regression model.

- sig and bonferroni indicate the significance level if the search algorithm selects eigenvectors based on their significance or the significance of residual spatial autocorrelation. If bonferroni=TRUE and objfn=' $p$ ', the significance level will be adjusted in order to account for inflated Type-I errors. If objfn= 'pMI ', bonferroni is automatically set to FALSE.

- positive (TRUE or FALSE) restricts the eigenvector search to those eigenvectors associated with positive levels of spatial autocorrelation.

- ideal. setsize (TRUE or FALSE) determines the ideal size of the candidate set $E^{C}$ according to the formula given in Chun et al. (2016). Note that this is only valid when filtering for positive spatial autocorrelation.

- alpha allows users to specify a threshold for the inclusion of eigenvectors in the candidate set based on their MC values (see Griffith, 2003).

- tol sets a tolerance threshold for remaining residual autocorrelation if objfn= ' MI '. Once the level of residual autocorrelation reaches the threshold, the selection procedure terminates.

- boot.MI (optional) takes integers indicating the number of bootstrap permutations in order to estimate the variance of the Moran test for residual autocorrelation.

These arguments allow users to customize the ESF model and obtain parameter estimates by using a single function call and only a few lines of code. While the print method for the output - an object of class "spfilter" - only reports the number of selected eigenvectors in $E^{*}$ and the size of the candidate set $E^{C}$, the summary method provides a host of useful additional information. 
R> (esf <- $1 m$ Filter $(y=y, x=X, W=W$, objfn="p", sig=. 1 , bonferroni=TRUE

$+\quad$,positive=TRUE, ideal. setsize=TRUE))

3 out of 22 candidate eigenvectors selected

$R>\operatorname{summary}(e s f, E V=T R U E)$

- Spatial Filtering with Eigenvectors (Linear Model) -

Coefficients (OLS):

Estimate $\quad S E \quad p$-value

(Intercept) $9.3708810 .712538324 .103548 \mathrm{e}-23 * * *$

$\begin{array}{llll}\text { beta_1 } & 0.975771 & 0.08536198 & 1.511830 \mathrm{e}-19 * * *\end{array}$

Adjusted R-squared:

Initial Filtered

0.46739450 .6534442

Filtered for positive spatial autocorrelation

3 out of 22 candidate eigenvectors selected

Objective Function: "p" (significance level=0.1)

Bonferroni correction: TRUE (adjusted significance level=0.00455)

Summary of selected eigenvectors:

Estimate SE p-value partialR2 VIF MI

$\begin{array}{llllllll}\text { ev_13 } & -9.552977 & 1.626696 & 6.290028 \mathrm{e}-08 & 0.23208263 & 1.005781 & 0.6302019 & * * *\end{array}$

$\begin{array}{lllllllll}\text { ev_10 } & -5.571465 & 1.632824 & 9.483754 \mathrm{e}-04 & 0.07901543 & 1.013360 & 0.7303271 & * * *\end{array}$

$\begin{array}{lllllllll}\text { ev_2 } & 4.900028 & 1.623316 & 3.261057 \mathrm{e}-03 & 0.06058390 & 1.001660 & 1.0004147 \quad * *\end{array}$

Moran's I ( Residuals):

Observed Expected Variance z p-value

Initial $0.3505680-0.01192610 \quad 0.01207299 \quad 3.299085 \quad 0.0004850019 * * *$

Filtered $0.1397003-0.03703186 \quad 0.02417938 \quad 1.1365620 .1278607838$

Besides the parameter estimates of the filtered model, the summary method provides information on the fit of the filtered and the unfiltered models, the objective function, and the Moran test for residual autocorrelation. If users specify EV=TRUE, information on the included eigenvectors in the order of their selection will be displayed as well. Just like above, we see that the eigenvector 13, for example, explains $23.21 \%$ of the variance, and the VIF indicates no problems of multicollinearity in the filtered model. The adjusted $R^{2}$ also shows that the ESF approach considerably improves model fit.
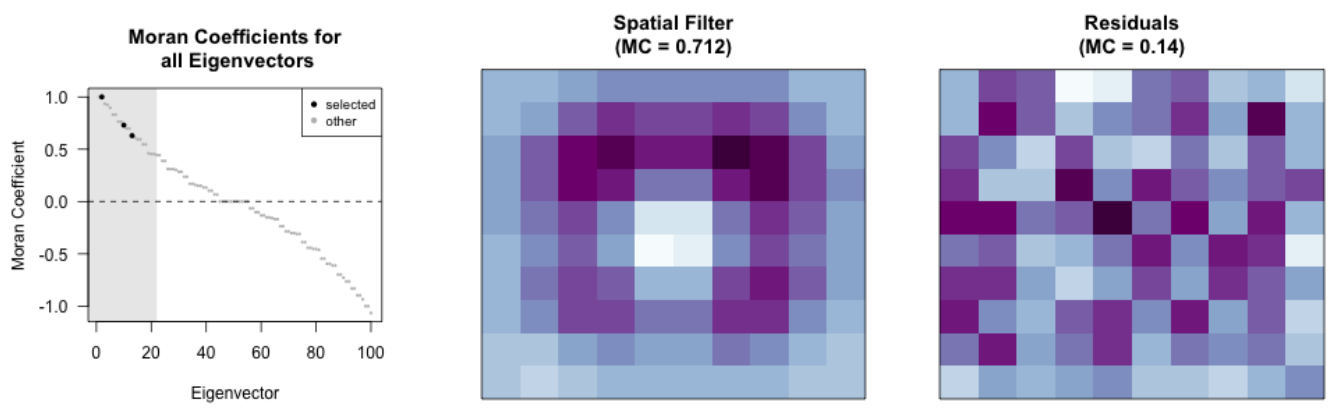

Figure 2: Plotting method for objects of class "spfilter" (left), spatial pattern captured by the filter and calculated by MI.sf() (center), and spatial patterns of filtered residuals (right).

Finally, the left part of Figure 2 demonstrates the plotting method for objects of class "spfilter" which is produced by plot (esf). It visualizes the MC of each eigenvector and highlights the ones selected by the unsupervised selection procedure. The grey shaded area illustrates the candidate set $E^{C}$ from which the eigenvectors in $E^{*}$ are selected. Figure 2 further depicts the spatial pattern of the spatial filter (center) and the filtered residuals (right). The function MI.sf() computes the MC value associated with the map pattern depicted by the spatial filter $E^{*} \gamma$ in Equation (3) (e.g., Le Gallo and Páez, 2013). 


\section{Spatial filtering in generalized linear models}

The ESF methodology directly extends to GLMs. In fact, one of the advantages of the filtering approach as compared to parametric spatial regression models in this context is that parameter estimates can be obtained by standard MLE and do not require the application of more sophisticated estimation techniques (Griffith et al., 2019).

Besides the supervised filtering procedure, the function $\operatorname{llmFilter}()$ from the spfilteR package allows users to perform unsupervised spatial filtering in GLMs. While its usage is purposefully similar to the function lmFilter() introduced above, GLMs require some adjustments of the filtering procedure. As a result, glmFilter () not only uses MLE instead of OLS to obtain parameter estimates but also differs in some of the function's input. Hence, in addition the input already discussed above, glmFilter() differs to lmFilter() with respect to the following input arguments:

- objfn defines the eigenvector selection criterium. Possible criteria are the maximization of model fit ('AIC' or 'BIC'), minimization of residual autocorrelation ('MI '), the significance level of candidate eigenvectors ( ' $\mathrm{p}$ '), the significance of residual spatial autocorrelation ( ' pMI '), or all eigenvectors in the candidate set ('all').

- model specifies the type of model to be estimated. The current version of spfilteR (version 1.0.0) supports 'probit', 'logit', and 'poisson' as input.

- optim.method determines the method used to optimize the likelihood function.

- min. reduction takes values in the interval $[0,1)$. It defines the minimum level of reduction in the AIC or BIC (if either selection criterium is chosen) relative to the current AIC/ BIC a candidate eigenvector needs to achieve in order to be included in the spatial filter.

- resid. type allows users to specify the type of residuals which is used to calculate the MC value. Valid arguments are 'raw', 'deviance', and the default option 'pearson'.

Implementing the ESF approach in GLMs using glmFilter() requires as few lines of code as using the lmFilter() function in the context of linear regression models. The following example demonstrates the ease of implementation in the context of a logit, a probit, and a Poisson regression model:

\# define DVs

$\mathrm{R}>\mathrm{y} \cdot \mathrm{bin}<-$ fakedataset\$indicator

$\mathrm{R}>\mathrm{y}$. count $<-$ fakedataset $\$$ count

\# seed (because of 'boot.MI')

set.seed(123)

\# logit model

R> (esf.logit <- glmFilter ( $y=y$.bin, $x=N U L L, W=W$, objfn="p", model="logit", optim.method="BFGS"

$+$ , sig=. 05 , bonferroni=FALSE, resid. type="pearson", boot. $\mathrm{MI}=100$ ))

3 out of 31 candidate eigenvectors selected

\# probit model

R> (esf.probit <- glmFilter ( $y=y$. bin, $x=N U L L, W=W$, objfn="BIC", model="probit"

$+\quad$,optim. method="BFGS", min. reduction= 0 , resid. type="deviance"

$+\quad$,boot.MI=100))

2 out of 31 candidate eigenvectors selected

\# poisson model

R> (esf. poisson <- glmFilter ( $y=y$. count, $x=N U L L, W=W$, objfn="pMI", model="poisson"

$+\quad$,optim.method="BFGS", sig=.1, resid.type="pearson"

$+\quad$, boot.MI=100))

0 out of 31 candidate eigenvectors selected

Of course, users can also define their own eigenvector selection criteria or apply the ESF approach to models currently not supported by the glmFilter () function. Just like for linear regression models illustrated above, the function getEVs () performs the eigenfunction decomposition of the transformed and symmetrized connectivity matrix, and users can implement a supervised selection procedure using the standard $g \operatorname{lm}()$ function. 


\section{A brief comparison to other $\mathbf{R}$ packages}

Of course, alternative implementations of the ESF approach outlined here exist in other R packages as well. While these packages are highly useful for spatial analysts, the spfilteR package offers a couple of notable extensions that improve these existing implementations. ${ }^{5}$

The spmoran package developed by Murakami (2020) contains different functions for estimating eigenvector-based spatial additive mixed models. Although the function esf() estimates a linear spatial filtering model, the main advantages of this package are the estimation of the random effects ESF model (e.g., Murakami and Griffith, 2019) and the fast approximation of the eigenfunction decomposition, which makes this package especially useful for large datasets. Moreover, users can also use the functions meigen() and meigen_f() to obtain eigenfunctions and perform supervised eigenvector selection.

At the same time, the eigenvector selection criteria implemented in esf () only allow for the identification of relevant eigenvectors based on model fit statistics such as the adjusted $R^{2}$, the AIC, or the BIC. The specification of the projection matrix $\boldsymbol{M}$ also does not allow for the inclusion of covariates. Furthermore, the spmoran package does not support the ESF approach in the context of GLMs.

Alternatively, the spatialreg package, which encompasses a great variety of different spatial estimation techniques, not only provides the Spatialfiltering() function estimating spatially filtered linear models. It also allows for the estimation of spatially filtered GLMs by using ME(). Yet, both of these functions utilize an objective function that selects eigenvectors based on the overall reduction of residual autocorrelation. While it is possible to restrict the candidate set size and to customize the level of remaining autocorrelation at which the search terminates, users cannot select alternative objective functions. Moreover, ME() does not allow for the inclusion of covariates in the construction of $\boldsymbol{M}$. Since there is no function to perform the eigenfunction decomposition shown in Equation (2), the package offers no support for supervised spatial filtering.

Therefore, the spfilteR package provides additional flexibility - especially for the estimation of filtered linear and generalized linear models where the ESF approach is predominantly applied. Since the eigenvector selection procedure is the crucial step in the ESF approach, the options provided by lmFilter() and glmFilter() allow users to tailor the ESF procedure to their specific needs. The option to estimate the ideal size of the eigenvector candidate set $E^{C}$ according to Chun et al. (2016), the specification of different residual types in GLMs, and the ability to define a threshold for the minimum increase in model fit when an objective function is chosen accordingly are examples of features unique to the spfilteR package.

Despite this additional flexibility, the functions that perform unsupervised eigenvector selection are very easy to use and only require a minimum of code. Moreover, the getEVs() command and several additional helper functions such as MI.ev(), MI.sf(), partialR2(), and vif.ev() introduced above facilitate the estimation of spatially filtered (generalized) linear models. Consequently, while the spmoran and the spatialreg packages cover additional model types and estimation strategies, the flexibility provided by the spfilteR package constitutes a great advantage in the most common applications of the ESF approach.

\section{Summary}

This article briefly covers the basics of spatial filtering with eigenvectors and introduces the spfilteR package. Using the synthetic dataset provided by the package, it discusses the main functions and their implementation in the context of supervised and unsupervised spatial filtering as well as its extension to GLMs. By comparing the package to alternative implementations of the ESF approach, this article highlights that the flexibility provided by the spfilteR package constitutes an important improvement in settings where the ESF approach is commonly applied.

\section{Acknowledgments}

Funding was provided by the German Research Foundation (DFG) through the Collaborative Research Center (SFB) 884 (grant number: 139943784). This work was also supported by a postdoc fellowship of the German Academic Exchange Service (DAAD).

\footnotetext{
${ }^{5}$ Note that other R packages such as adespatial (Dray et al., 2020) and vegan (Oksanen et al., 2019) also provide tools to eigendecompose a transformed connectivity matrix and implement the supervised ESF approach. Yet, since these packages are not primarily concerned with ESF and do not support unsupervised spatial filtering, I do not discuss them in greater detail here.
} 


\section{Bibliography}

R. Bivand and G. Piras. Comparing implementations of estimation methods for spatial econometrics. Journal of Statistical Software, 63(18):1-36, 2015. URL https://www. jstatsoft. org/v63/i18/. [p450]

Y. Chun and D. A. Griffith. A quality assessment of eigenvector spatial filtering based parameter estimates for the normal probability model. Spatial Statistics, 10:1-11, 2014. URL https://doi .org/ 10.1016/j.spasta.2014.04.001. [p451]

Y. Chun, D. A. Griffith, M. Lee, and P. Sinha. Eigenvector selection with stepwise regression techniques to construct eigenvector spatial filters. Journal of Geographical Systems, 18(1):67-85, 2016. URL https://doi.org/10.1007/s10109-015-0225-3. [p451,454,457]

A. D. Cliff and J. K. Ord. Testing for spatial autocorrelation among regression residuals. Geographical Analysis, 4(3):267-284, 1972. URL https: //doi .org/10.1111/j. 1538-4632.1972. tb00475.x. [p450]

A. D. Cliff and J. K. Ord. Spatial Processes: Models E Applications. Advances in Spatial Science. Pion, London, 1981. ISBN 9780850860818. [p450, 452]

D. Darmofal. Spatial Analysis for the Social Sciences. Analytical Methods for Social Research. Cambridge University Press, New York, USA, 2015. URL https: //doi .org/10. 1017/CB09781139051293. [p450]

S. Dray, D. Bauman, G. Blanchet, D. Borcard, S. Clappe, G. Guenard, T. Jombart, G. Larocque, P. Legendre, N. Madi, and H. H. Wagner. adespatial: Multivariate Multiscale Spatial Analysis, 2020. URL https: //CRAN.R-project.org/package=adespatial. R package version 0.3-8. [p457]

R. J. Franzese and J. C. Hays. Spatial econometric models of cross-sectional interdependence in political science panel and time-series-cross-section data. Political Analysis, 15(2):140-164, 2007. URL https://doi.org/10.1093/pan/mpm005. [p450]

A. Getis and D. A. Griffith. Comparative spatial filtering in regression analysis. Geographical Analysis, 34(2):130-140, 2002. URL https://doi .org/10.1111/j.1538-4632.2002. tb01080.x. [p450]

M. F. Goodchild. What problem? spatial autocorrelation and geographic information science. Geographical Analysis, 41(4):411-417, 2009. URL https://doi.org/10.1111/j.1538-4632.2009.00769.x. [p450]

D. Griffith and C. G. Amrhein. Multivariate Statistical Analysis for Geographers. Prentice Hall, Englewood Cliffs, 1997. ISBN 9780136056928. [p451]

D. Griffith and Y. Chun. Spatial autocorrelation and spatial filtering. In M. M. Fischer and P. Nijkamp, editors, Handbook of Regional Science, pages 1477-1507. Springer, Berlin, Heidelberg, 2014. URL https://doi.org/10.1007/978-3-642-23430-9_72. [p451,452]

D. A. Griffith. Spatial autocorrelation and eigenfunctions of the geographic weights matrix accompanying geo-referenced data. The Canadian Geographer, 40(4):351-367, 1996. URL https: //doi.org/10.1111/j.1541-0064.1996.tb00462.x. [p450, 451, 453]

D. A. Griffith. A linear regression solution to the spatial autocorrelation problem. Journal of Geographical Systems, 2(2):141-156, 2000. URL https: //doi.org/10.1007/PL00011451. [p450]

D. A. Griffith. Spatial Autocorrelation and Spatial Filtering: Gaining Understanding Through Theory and Scientific Visualization. Advances in Spatial Science. Springer, Berlin, Heidelberg, 2003. URL https: //doi.org/10.1007/978-3-540-24806-4. [p450, 451, 452, 453, 454]

D. A. Griffith, Y. Chun, and B. Li. Spatial Regression Analysis Using Eigenvector Spatial Filtering. Spatial Econometrics and Spatial Statistics. Elsevier, London, 2019. ISBN 9780128150436. URL https: //doi.org/10.1016/B978-0-12-815043-6.09990-0. [p452,456]

J. Le Gallo and A. Páez. Using synthetic variables in instrumental variable estimation of spatial series models. Environment and Planning A: Economy and Space, 45(9):2227-2242, 2013. URL https: //doi.org/10.1068/a45443. [p452, 455]

D. Murakami. spmoran: Moran Eigenvector-Based Scalable Spatial Additive Mixed Modeling, 2020. URL https://CRAN.R-project.org/package=spmoran. R package version 0.2.0-2. [p450, 457]

D. Murakami and D. A. Griffith. Eigenvector spatial filtering for large data sets: Fixed and random effects approaches. Geographical Analysis, 51(1):23-49, 2019. URL https://doi org/10.1111/gean. 12156. [p457] 
J. Oksanen, F. G. Blanchet, M. Friendly, R. Kindt, P. Legendre, D. McGlinn, P. R. Minchin, R. B. O'Hara, G. L. Simpson, P. Solymos, M. H. H. Stevens, E. Szoecs, and H. H. Wagner. vegan: Community Ecology Package, 2019. URL https://CRAN. R-project. org/package=vegan. R package version 2.5-6. [p457]

A. Páez. Using spatial filters and exploratory data analysis to enhance regression models of spatial data. Geographical Analysis, 51(3):314-338, 2019. URL https://doi .org/10.1111/gean . 12180. [p452]

M. Tiefelsdorf and B. Boots. The exact distribution of moran's i. Environment and Planning A: Economy and Space, 27(6):985-999, 1995. URL https://doi .org/10.1068/a270985. [p451,453]

M. Tiefelsdorf and D. A. Griffith. Semiparametric filtering of spatial autocorrelation: The eigenvector approach. Environment and Planning A: Economy and Space, 39(5):1193-1221, 2007. URL https: //doi.org/10.1068/a37378. [p450, 451, 452, 454]

Sebastian Juhl

Collaborative Research Center 884

University of Mannheim

B6, 30-32

68159 Mannheim

Germany

ORCiD: https://orcid.org/0000-0002-7123-5398

sebastian. juhl@gess. uni-mannheim. de 